\section{Marburger Liquor-Modell}

T. O. Kleine

Institut für Laboratoriumsmedizin und Pathobiochemie, Molekulare Diagnostik Standort Marburg Referenzlabor für Liquordiagnostik, UKGM Universitätsklinikum Gießen und Marburg, Marburg, Deutschland

Synonym(e) Physiologie von Cerebrospinalflüssigkeit (CSF)

Englischer Begriff Marburg model for formation and diagnostics of human cerebrospinal fluid

Definition Cerebrospinalflüssigkeit (CSF) ist eine Mischung aus Ventrikelliquor (V-CSF), Subokzipitalliquor (SOP-CSF) und Lumballiquor (Lumbal-CSF).

Beschreibung V-CSF in 2 Seitenventrikel mit $\leq 1$ Leukozyt/ $\mu 1$, zurückgedrängt aus Ventrikel V3 (weniger V4) mit $\leq 3$ Leukozyten/ $\mu 1$, gepresst durch 6 zirkumventrikuläre Organe (CVOs) ( $\triangleright$ Liquor cerebrospinalis) aus Blut in V-CSF: kleine lymphozytäre Zellen $>>$ größere monozytäre Zellen ( $\triangleright$ Liquor-Monozyten); V-CSF in V3 und V4 fließt in SOPLiquor und hauptsächlich kranial in Schädelkalotte, um in Arachnoidvilli resorbiert zu werden. Nur ein Teil SOP-CSF gelangt in den Spinalraum und wird modifiziert durch Flüssigkeitsabfluss in Nervenscheiden der Spinalnerven. In Lumbal-CSF fließt etwas Lymphe aus Ductus thoracicus zurück und erhöht die Leukozytenzahl auf $\leq 5$ Leukozyten $/ \mu 1$ (๖ Liquor-Zellzählung, mikroskopisch).

Das Marburg Liquor-Modell erklärt variierende Proteinkonzentration in CSF: In V-CSF $50-180 \mathrm{mg} / \mathrm{L}$, in SOP-CSF 133-267 mg/L Gesamtprotein: Blut-Plasma, filtriert durch Molekularsieb in „tight junctions“ von Choroidepithel von 4 Plexus choroidei, lokalisiert in V1, V2, V3, V4 (Blutkapillaren in Choroidplexus ohne „tight junctions“ im Gegensatz zu ZNS-Kapillaren); filtrierte Blutproteine werden gemischt mit Lymphproteinen aus Ductus thoracicus auf 209-412 mg/L Gesamtprotein in Lumbal-CSF ( $\triangleright$ LiquorProtein).

Variierende Einflussgrößen sind: Blutdruck für Blutleukozyten und Plasmaproteine; Körperhaltung (Stehen, Liegen), tief Atmen, Pressen, Husten, Nießen u. a. m. für Lymphrückfluss aus Ductus thoracicus.

Stichworte, auf die verwiesen wird:

$\checkmark$ Liquor cerebrospinalis

- Liquor-Monozyten

• Liquor-Zellzählung, mikroskopisch

$\checkmark$ Liquor-Protein

\section{Literatur}

Kleine TO (2015) Cellular immune surveillance of central nervous system bypasses blood-brain barrier and blood-cerebrospinal-fluid barrier: revealed with the New Marburg Cerebrospinal-Fluid Model in healthy humans. Cytometry A 87:227-243 